\title{
Development of a 3D Body Database to Improve Measures of Perceptual Male Body Image Distortion
}

\author{
Sophie MOHAMED ${ }^{* 1,2}$, Nadia MAALIN¹, Robin KRAMER ${ }^{1}$, \\ Andrew IRVINE ${ }^{1}$, Piers L. CORNELISSEN ${ }^{3}$, Martin J. TOVÉE ${ }^{1}$ \\ ${ }^{1}$ School of Psychology, University of Lincoln, UK; \\ ${ }^{2}$ Lincoln Institute for Health, University of Lincoln, UK; \\ ${ }^{3}$ School of Psychology, Northumbria University, UK
}

\begin{abstract}
https://doi.org/10.15221/19.173

There are important limitations in the current methodological stimuli being used in perceptual body image research among men, including a reliance on unrealistic images, a lack of biometric validity, and little consideration of the sexual dimorphism of human body composition. This research seeks to address this critical 'stimulus problem' through the development of a large database of 3D body scans and body composition measurements. The aim of this study is to investigate the relationship between body shape and composition, as well as how this varies within distinct body mass index (BMI) categories. Over 170 adult males have been recruited through staff and students at the University of Lincoln and from the general population. Principal component analysis and partial least squares regression are being used to characterise the statistical mapping between 3D male body shape changes and body composition. These statistical models will then allow for the development of a range of photorealistic, biometrically accurate, calibrated 3D male avatars, with future applications in both healthcare and research contexts.
\end{abstract}

Keywords: Male body image, perception, 3D body scanning, muscularity, adiposity, body size estimation

\section{Introduction}

Perceptual body image distortion is characterised by a discrepancy between a person's perceived and actual body shape and size, and this cognitive misinterpretation is associated with negative physical and psychological health outcomes. Historically, a variety of methodologies have been applied to assess perceptual body image in both general and clinical populations, including figure drawing scales, eye-tracking and video distortion techniques. Figure drawing scales and other two-dimensional (2D) sets of photographs have several methodological limitations. These scales are often limited in their realism and only present partial variability in shape changes between figures [1]. In addition, presenting figures in 2D limits the richness of body shape and size information available to participants and is unlikely to include an accurate representation of an individual's specific current or ideal body perceptions.

In response to these limitations, interactive three-dimensional (3D) computer programs have been developed more recently to measure body image perceptions using a method of adjustment. However, methods of adjustment can also be problematic in that the original size of the body presented to participants can have a significant impact on participants' responses [2]. In addition, they often only measure an estimate of the participant's perceived overall body size, and do not consider the perceived size or shape of individual parts of the body. More updated software has since been developed, with ongoing improvements in technology, that allow participants to alter realistic virtual 3D bodies in a large variety of dimensions to create specific, detailed changes in body size and shape. 3D body scanning technologies have also become a popular and increasingly accessible method of understanding body image, as well as measuring actual individual variation in body size and shape. 3D body scanning is now being used in a variety of research looking at variation in human body shape [3, 4], obesity [5], and body shape morphing across individuals [3].

Additional methodological limitations stem from a historical focus of research on the female population. Sex differences in human body composition from birth can lead to different patterns of physical development throughout the lifespan, which in turn result in the formation of different body statures and shapes between men and women [6]. For example, males have a greater proportion of lean mass from infancy and their body shape tends to remain relatively constant over the lifespan, while females tend to experience an upward shift in their weight as they age, from their thighs to their bust and waist $[6,7$, 8]. This sexual dimorphism can be explained using an evolutionary perspective, where larger male size is related to male-to-male competition and is used as a judgement of health and reproductive potential by females [1].

*sMohamed@lincoln.ac.uk 
Additional methodological limitations stem from a historical focus of research on the female population. Sex differences in human body composition from birth can lead to different patterns of physical development throughout the lifespan, which in turn result in the formation of different body statures and shapes between men and women [6]. For example, males have a greater proportion of lean mass from infancy and their body shape tends to remain relatively constant over the lifespan, while females tend to experience an upward shift in their weight as they age, from their thighs to their bust and waist $[6,7$, 8]. This sexual dimorphism can be explained using an evolutionary perspective, where larger male size is related to male-to-male competition and is used as a judgement of health and reproductive potential by females [1].

Within both research and healthcare settings, body mass index (BMI) is widely used as a measure of health and nutritional status, as well as an indicator of the risks related to increased body weight. Alternatively, there has been a recent focus on abdominal fat as an indicator of health, due to its association with increased disease risk and mortality [6, 9]. Abdominal fat is often assessed through the measurement of body girths such as waist circumference and the waist-to-hip ratio (WHR). The WHR may also be a better indicator of general body shape than BMI, as BMl's focus on height and weight can result in men with larger muscle mass being classified as overweight or obese.

Much more than is the case for women, male body shape is derived from a complex interaction between adiposity and muscle mass. Therefore, whereas stimuli for use with female populations may only vary in adiposity, those for use with males need to vary in a number of different dimensions. Therefore, there is a critical need to develop and validate more biometrically accurate, ecologically valid stimuli for use in male perceptual body image research, that account for male-specific body composition and realistic patterns of body shape development.

\section{Method}

\subsection{Participants}

171 adult males, aged between 18 and 45 , have been recruited through staff and students at the University of Lincoln and from the general public. Inclusion criteria for participation included not having a current or previous history of an eating or body image disorder. Participants with a pacemaker or electrical implant, photosensitive epilepsy or individuals prone to migraines were not permitted to take part.

\subsection{Study Procedure}

During each session, participants self-reported demographic information, including their age, ethnicity and sex. High resolution, colour 3D body scans of each participant were obtained using a 24 SLR camera 3DMd anthropometric surface imaging system. The 3D scanner contains nine modular camera units (MCU), with each MCU comprising of two mono cameras, a speckle projector and a colour camera, and scans at 7 frames per second. The speckle camera turns on when the mono cameras are taking an image, while light emitting diode (LED) panels are turned on when the colour camera is taking an image. Participants were required to stand in the centre of the scanner and slowly raise their arms by their sides, with their legs shoulder-width apart, for 20 seconds. Participants were provided with grey 'jersey trunks' to wear during the scan. Measurements of body composition were then taken using a Tanita MC-780MA body composition analyser. This scale uses a small undetectable electrical current to accurately measure each participant's fat mass, fat free mass, skeletal muscle mass, water content, visceral fat, BMI, and basal metabolic rate (BMR). Lastly, physical body measurements were taken for each participant, including height $(\mathrm{cm})$, weight $(\mathrm{kg})$, chest circumference $(\mathrm{cm})$, hip circumference $(\mathrm{cm})$, waist circumference $(\mathrm{cm})$ and bicep circumference $(\mathrm{cm})$.

\subsection{Data Analysis}

The 3D scans have been processed using Wrap3 software (Wrap 3.3.17, Russian3DScanner, 2018), to repair or remove any non-manifold topology and small irrelevant components from each individual scan. A standard base mesh was wrapped around each scan by matching corresponding control points on features of both models. Polygon selection was also used to exclude the hands in each scan from wrapping, as this feature is not relevant to the data analysis.

Principal component analysis (PCA) and multivariate partial least squares (PLS) regression are currently being used to characterise the statistical mapping between 3D male body shape changes and body composition, with a particular focus on muscle and adiposity. This has involved removing the head, hands and feet from the 3D meshes, as these parts of the body are not of interest. Preliminary PCA with a subset of the scans highlighted large variation in the bodies, particularly in regards to height, body posture, and arm and leg positioning. Therefore, PCA is now being run with the 3D scans segmented into the arms, legs and torso, and with each segment centred to the same point of origin. 


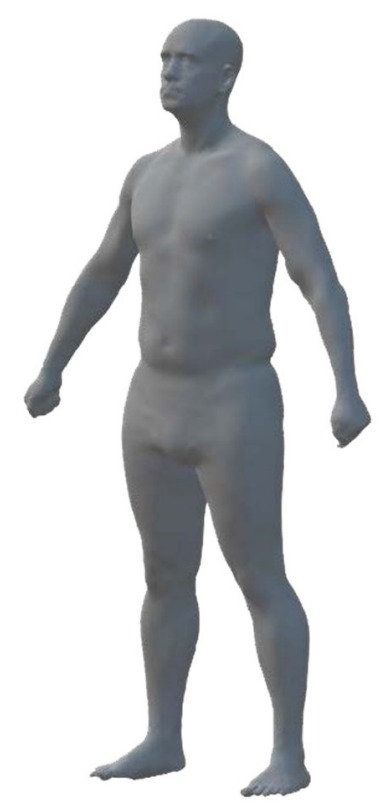

Fig 1. Example of a processed $3 D$ body scan.

\section{Discussion}

The development of new biometrically accurate measures of male body shape and size estimations may have implications for clinical and healthy male populations, both within a research and health context. Clinically, this resource could be used in the development of interventions to treat distorted body image in males, for the use of health professionals in informing conversations and working with overweight, obese and eating disorder patients, as well as for monitoring the progress of healthy weight maintenance efforts. Additionally, it may have applications in research to improve the accuracy and specificity of estimates relating to body shape and size of the self or of a third party, where the observer can visually match for perceived or ideal adiposity, muscle mass and visual muscle tone. It may also be useful for exploring social perceptions and visual judgements of body shape, weight and muscularity. As a whole, the data collected has produced a database of systematic male measurements that can be used in further body image research independently or in conjunction with already existing databanks.

\section{References}

[1] K. L. Crossley, P. L. Cornelissen, and M. J. Tovée, "What is an attractive body? Using an interactive 3D program to create the ideal body for you and your partner," PLoS One, vol. 7, no. 11, p. e50601, 2012.

[2] C. T. Fuentes, M. R. Longo, and P. Haggard, "Body image distortions in healthy adults," Acta psychologica, vol. 144, no. 2, pp. 344-351, 2013.

[3] B. Allen, B. Curless, B. Curless, and Z. Popović, "The space of human body shapes: reconstruction and parameterization from range scans," in ACM transactions on graphics (TOG), 2003, vol. 22, no. 3: ACM, pp. 587-594.

[4] Z. B. Azouz, M. Rioux, C. Shu, and R. Lepage, "Characterizing human shape variation using 3D anthropometric data," The visual computer, vol. 22, no. 5, pp. 302-314, 2006.

[5] J. Wells, A. Ruto, and P. Treleaven, "Whole-body three-dimensional photonic scanning: a new technique for obesity research and clinical practice," International Journal of Obesity, vol. 32, no. 2, p. 232, 2008.

[6] J. C. Wells, "Sexual dimorphism of body composition," Best practice \& research Clinical endocrinology \& metabolism, vol. 21, no. 3, pp. 415-430, 2007.

[7] S. J. Fomon, F. Haschke, E. E. Ziegler, and S. E. Nelson, "Body composition of reference children from birth to age 10 years," The American journal of clinical nutrition, vol. 35, no. 5, pp. 1169-1175, 1982.

[8] N. C. de Bruin et al., "Standards for total body fat and fat-free mass in infants," Archives of disease in childhood, vol. 74, no. 5, pp. 386-399, 1996.

[9] M. Ashwell, P. Gunn, and S. Gibson, "Waist-to-height ratio is a better screening tool than waist circumference and BMI for adult cardiometabolic risk factors: systematic review and metaanalysis," Obesity reviews, vol. 13, no. 3, pp. 275-286, 2012. 